\title{
P03-13-23 Poster session
}

\section{Modulation of unfolded protein response by methylmercury ( $\mathrm{MeHg}$ )}

Hideki Hiraoka ${ }^{1}$, Kengo Nakahara ${ }^{1}$, Kosaku Okuda ${ }^{1}$, Yoshito Kumagai ${ }^{2}$, Takashi Uehara ${ }^{1}$

${ }^{I}$ Department of Medicinal Pharmacology, Graduate School of Medicine, Dentistry, and Pharmaceutical Sciences, Okayama University, Japan, ${ }^{2}$ Doctoral Program in Biomedical Sciences, Graduate School of Comprehensive Human Sciences, University of Tsukuba, Japan

\section{[Background]}

MeHg known as a causative factor of Minamata disease induces neuronal cell death and damage the central nervous system, but the mechanism is poorly understood. Previously, we reported that MeHg induces S-mercuration at cysteine 383 or 386 in protein disulfide isomerase (PDI), and this modification induces the loss of enzymatic activity. Because PDI is a key enzyme for the maturation of nascent protein harboring a disulfide bond, the disruption in PDI function by $\mathrm{MeHg}$ results in endoplasmic reticulum (ER) stress via the accumulation of misfolded proteins. However, the effects of $\mathrm{MeHg}$ on unfolded protein response (UPR) sensors remain unclear. In the present study, we examined whether UPR is regulated by MeHg.

[Methods]

To investigate the activations of UPR sensors and downstream signaling by MeHg stimulation, we used western blot analysis and RT-PCR. To measure apoptosis cells induced by MeHg, we assessed the chromosomal condensation using the fluorescent dye Hoechst 33342.

[Results]

We found that $\mathrm{MeHg}$ activates protein kinase RNA-like endoplasmic reticulum kinase (PERK) and activating transcriptional factor 6 (ATF6) branches. Although phosphorylated inositol-requiring enzyme $1 \alpha$ (IRE1 $\alpha$ ) was detected, $\mathrm{MeHg}$ did not induce the cytosolic splicing of immature $\mathrm{x}$-box binding protein 1 (XBP1) mRNA (a selective substrate of IRE1 $\alpha$ ). Then, the IRE1 $\alpha$-null MEFs were transfected with vectors encoding wild-type hemagglutinin (HA)-tagged IRE1 $\alpha$ or cysteine mutants. MeHg-induced inhibition of IRE1 $\alpha$ endonuclease activity was significantly ameliorated in the MEFs that expressed IRE1 $\alpha$ (C931S). These results suggested that C931 in IRE1 $\alpha$ could be a predominant target of MeHg. A previous study has shown that the IRE1 $\alpha$-XBP1 branch functions as an anti-apoptotic pathway. In contrast, the PERK/ATF6 branches are involved in the induction of cell death. Therefore, these signals may be implicated in the MeHg-induced loss of cell viability. Indeed, treatment with GSK2606414, a specific PERK inhibitor, significantly attenuated MeHg-induced cell death.

[Conclusions]

We demonstrated that MeHg disrupts anti-apoptotic signaling based on the IRE1 $\alpha$-XBP1 branch and simultaneously promotes pro-apoptotic signaling via the PERK/ATF6 branches. These findings may be utilized in the development of novel therapeutic approaches for Minamata disease. 